\title{
Atraumatic Restorative Treatment-Sealed versus Nonsealed First Permanent Molars: A 3-Year Split-Mouth Clinical Trial
}

\author{
Daniela Hesse ${ }^{a}$ Camila de Almeida Brandão Guglielmi ${ }^{\text {b }}$ \\ Daniela Prócida Raggio ${ }^{b}$ Marcelo José Strazzeri Bönecker ${ }^{b}$ \\ Fausto Medeiros Mendes ${ }^{b}$ Clarissa Calil Bonifácio $^{a}$ \\ a Department of Pediatric Dentistry, Academic Center for Dentistry Amsterdam, Amsterdam, The Netherlands; \\ ${ }^{b}$ Department of Orthodontics and Pediatric Dentistry, Dental School, University of São Paulo, São Paulo, Brazil
}

\section{Keywords}

Dental caries · Glass ionomer cements · Pit and fissure sealants · Prevention

\begin{abstract}
To investigate the efficacy of atraumatic restorative treatment (ART) sealants vs. no sealant in preventing the development of dentine caries lesions in first permanent molars over a period of 3 years. A total of 187 schoolchildren (aged 6-8 years) from a low-income population presenting the 4 first permanent molars without clinically detectable dentine caries lesions were selected to be part of a split-mouth clinical trial. All 4 first permanent molars were investigated in this trial and the children's mouth was split vertically into left and right sides; therefore, 2 molars were randomly allocated to receive ART sealants, while the other 2 molars remained nonsealed. All children received toothbrushing instructions and dietary advice every 6 months for a period of 3 years. Clinical evaluations were performed after 3, 6, 12, 18, 24, and 36 months and both sealant retention and dental caries were scored. Kaplan-Meier survival analysis, log-rank test, and Cox regression with shared frailty analysis were performed. A cavitated dentine caries lesion was considered a failure. The
\end{abstract}

cumulative survival rates of dentine cavity-free first permanent molars were $90 \%$ for ART-sealed molars and $90.8 \%$ for nonsealed molars, with no statistically significantly difference between sealed and nonsealed molars $(p=0.70)$. The retention of sealants was not associated with the development of cavitated dentine caries and children presenting a higher baseline caries experience had greater chances of developing dentine lesions. In conclusion, the application of ART sealants was not more efficacious than nonsealing in reducing the development of dentine cavitated lesions in first permanent molars.

(C) 2020 The Author(s)

Published by S. Karger AG, Basel

\section{Introduction}

The permanent molars of children and adolescents are often affected by caries lesions, and the occlusal surfaces are particularly at risk [Mejàre et al., 1998]. The reason for this high susceptibility lies on the fact that dental caries is a biofilm-dependent disease and, although this relationship between biofilm and dental caries has already been well established, discussions still exist regarding the structural composition of biofilms and their cariogenicity

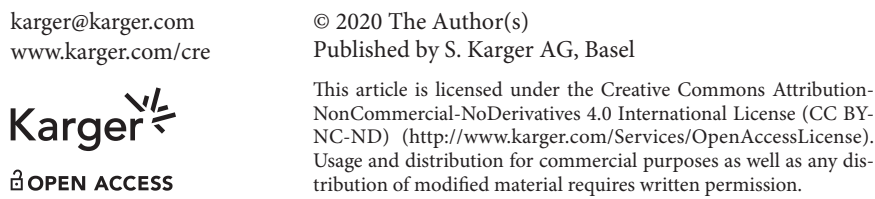

karger@karger.com www.karger.com/cre

Karger $\stackrel{\text { ' }}{=}$

BOPEN ACCESS

(C) 2020 The Author(s)

Published by S. Karger AG, Basel

This article is licensed under the Creative Commons AttributionNonCommercial-NoDerivatives 4.0 International License (CC BYNC-ND) (http://www.karger.com/Services/OpenAccessLicense). Usage and distribution for commercial purposes as well as any distribution of modified material requires written permission.

Daniela Hesse

Department of Pediatric Dentistry, Academic Center for Dentistry Amsterdam (ACTA) Gustav Malheuren 3004

NL-1081 LA Amsterdam (The Netherlands)

d.hesse@acta.nl 
[Carvalho et al., 2016]. In fact, it has been discussed that the presence of cariogenic microorganisms does not always lead to dental caries development and this disease actually occurs when a disequilibrium among the biofilm, the host, and the microenvironment happens [Colombo and Tanner, 2019]. For occlusal surfaces, it has been stated that the dental anatomy, as well as the position of permanent molars in the mouth, hinders effective toothbrushing by children, thereby facilitating plaque accumulation [Carvalho et al., 1992] and, as a result of the disequilibrium of the factors already named, caries lesions can develop.

Toothbrushing is a self-care behavior essential for removal of the biofilm and maintenance of oral health [Poklepovic et al., 2013] and it should be enough to keep individuals in a healthy condition. However, patients often experience difficulties achieving adequate biofilm control [Choo et al., 2001] and additional measures can be required to avoid the development of caries lesions. For instance, intensive educational care supplemented by professional cleaning and fluoride application during the eruption period of permanent molars has been demonstrated to be an effective measure to conserve the integrity of occlusal surfaces [Carvalho et al., 1992]. Also, it has been suggested that supervised toothbrushing combined with fluoride-containing toothpaste is capable of decreasing the caries incidence in permanent molars [Jackson et al., 2005]; however, a recent systematic review concluded that the magnitude of the benefits resulted from supervised toothbrushing could not be measured and no conclusions about the effectiveness of this intervention could be drawn [Dos Santos et al., 2018].

Another caries-preventive measure is the use of pit and fissure sealants. These sealants were developed to be applied on the occlusal surface of caries-prone teeth, preventing plaque stagnation on those areas [AhovuoSaloranta et al., 2017]. Actually, the use of sealants is an effective approach in preventing dental caries and, when it comes to the type of sealant, although the retention rate of resin-based sealants has been reported to be higher than that of glass ionomer cement (GIC)-based sealants, the caries preventive effect of both materials have been reported to be similar [Mickenautsch and Yengopal, 2016]. In fact, 2 systematic reviews showed that GIC sealants applied following the atraumatic restorative treatment (ART) approach can be effective in preventing caries lesions development, and it can be used as a preventive measure as well as in the treatment of incipient lesions [van't Hof et al., 2006; de Amorim et al., 2012].

Caries-Preventive Effect of ART Sealants
When it comes to a direct comparison between ART sealants and toothbrushing, an investigation showed that children that received ART sealants on the permanent molars presented a lower caries increment compared to those who followed a school-based toothbrushing program or those who received no intervention; however this research presents some methodological limitations that unfortunately lower the level of evidence of the findings and the results should be interpreted with caution [Monse et al., 2012]. Recently, Hilgert et al. [2015] investigated the caries-preventive effect of daily supervised toothbrushing at schools compared to resin-based and ART sealants on permanent molars. In that study, a surface-level risk assessment to determine the caries risk of erupted first permanent molars was performed. Occlusal surfaces presenting ICDAS codes 2 and 3 or a combination of ICDAS code 1 and medium or deep fissures were considered high-caries-risk surfaces, while those presenting ICDAS codes 0 or 1 and shallow fissures were considered lowcaries-risk surfaces. In this way, an accurate comparison between treatments for surfaces presenting a high or low caries risk was allowed, instead of the child-level caries risk assessment. Additionally, the surface-level risk assessment grouped the same stages of caries lesion development at baseline, avoiding comparison of surfaces that at the beginning had no signs of a caries lesion with those that already presented an initial caries lesion, a situation that is often seen in this type of study. The high-cariesrisk occlusal surfaces were allocated to receive either daily supervised toothbrushing or resin-based or ART sealants, while the low-risk surfaces were allocated to receiving daily supervised toothbrushing or no intervention. The results showed that approximately $90 \%$ of the first permanent molars did not develop caries lesions in dentine after 3 years of evaluation, and no statistically significant difference was found between the 3 groups for high-caries-risk occlusal surfaces and, as may be expected for low-risk surfaces, no effect of the intervention was observed. After that, Goldman et al. [2017] published the results of the economic analysis of these 3 strategies and they found that the costs involved in daily supervised toothbrushing was at least 2.5 times higher than those of sealant application, which could impair the implementation of this daily based preventive program, especially in socioeconomically deprived populations.

Although it has been discussed that the application of pit and fissure sealants should be linked to an individual and detailed caries risk assessment, including parameters such as caries experience, caries activity, and the tooth's erupting stage and anatomy [Splieth et al., 2010], sealants 
are still used indiscriminately as the main tool for prevention and management of occlusal caries lesions in populations or areas with a low socioeconomic status [Griffin et al., 2016]. In this way, we aimed to investigate whether the application of an ART sealant is more effective in preventing cavitated dentine caries lesions compared to nonsealing in first permanent molars of schoolchildren from a low socioeconomic area. As part of study focused on the longevity of ART sealants applied on first permanent molars [Hesse et al., 2015], teeth were also evaluated regarding the development of cavitated dentine caries lesions. The current article comprises an analysis regarding the efficacy of ART sealants in preventing cavitated dentine caries lesions compared to nonsealed first permanent molars over a 3-years period.

\section{Materials and Methods}

\section{Sampling Procedure and Study Design}

This study is a split-mouth clinical trial that was carried out between March 2010 and June 2013, and it was reported following the CONSORT guidelines for reporting within person randomized trials [Pandis et al., 2017]. Children were treated and evaluated in 26 public schools of Barueri, a city located in Brazil's southeast region whose water system is artificially fluoridated $(0.7 \mathrm{mg} / \mathrm{L})$. The sample size calculation considered the primary outcome to be the "retention rate of 2 different high viscous ART sealants" and the 1-year results were published elsewhere [Hesse et al., 2015]. For sample size estimation, a two-tailed test was used based on the 3 -year retention rate of ART sealants (72\%) [de Amorim et al., 2012]. A minimally important difference of $20 \%$ in success rates between the 2 GICs was assumed, and an $\alpha$ of $5 \%$ and a power of $80 \%$ were considered. After an increase of $20 \%$ to compensate possible losses to follow-up, a final sample size of 162 children was required.

This study was approved by the Local Research Ethics Committee (protocol 190/08; School of Dentistry, University of São Paulo, Brazil). Parents and/or legal guardians were informed about the investigation and treatments and written consent was obtained. Initially, 2,000 schoolchildren aged 6-8 years were screened. A total of 187 children who fulfilled the inclusion criteria were included in the research. The inclusion criteria were: (1) children with cooperative behavior and in good physical and medical health; (2) children who presented a signed consent form; (3) the presence of 4 first permanent molars without gingival tissue covering the occlusal surface; and (4) 4 first permanent molars without clinically detectable cavitated enamel or dentine caries lesions, evaluated using ICDAS criteria (scores 0, 1, and 2) [Ismail et al., 2007].

This study is registered at ClinicalTrials.gov (Atraumatic Restorative Treatment Sealants in First Permanent Molars; NCT03667768).

\section{Implementation}

Four final-year undergraduate dental students previously trained and calibrated in the ICDAS criteria [Ismail et al., 2007] for the assessment of caries, and in the Nyvad criteria [Nyvad et al.,
1999] for the assessment of lesion activity, were responsible for the children's baseline evaluation. The training process comprised $4 \mathrm{~h}$ of specific training, and calibration sessions with a "benchmark examiner," using clinical slides. Afterward, hands-on training comprised of evaluation of 30 molars was carried out. The $\kappa$ coefficient values ranged from 0.85 to 0.94 and from 0.70 to 0.90 for inter- and intra-examiner consistency tests, respectively.

The operators were also trained in how to apply ART sealants [Frencken et al., 1996] by clinicians experienced in this treatment modality as well as in clinical trials. First a lecture about ART was given. After that, the undergraduate students participated in a hands-on laboratory-based workshop for handling and application of the GICs used in this trial, as well as for the application of ART sealants in extracted molars. A try-out week with children was included for familiarization of operators with the local conditions, caries lesions detection, and sealant application. During this week, 30 children who were not included in the final sample were treated. The operators were divided into 2 pairs and were assisted by each other. All sealants were applied on school premises without the use a dental chair or other facilities from a dental office. A headlight was used to aid the detection of lesions and the application of sealants. Also, gauze, cotton wool rolls, and a manual syringe were used to dry the teeth for caries detection.

\section{Intervention}

In this research, no individual caries risk assessment was carried out; however, the included children belonged to a low-income population with limited access to health care. Thus, we assumed that those children could be considered at high-caries-risk for development of caries. All 4 first permanent molars of the selected children were included in this investigation. The children's mouth was split vertically into left and right sides, and 2 molars were randomly allocated to receive ART sealants with either one (Fuji IX; GC Europe, Leuven, Belgium) or another brand of GIC (Maxxion; FGM, Joinvile, Brazil), and the other 2 molars remained nonsealed. The randomization procedure was done with the aid of 2 computer-generated random lists. The first one was used to assign the side of the mouth to be treated, while the second one was used to assign the children to the GIC brands used in this study. Four independent dentists who work in the municipality were invited to carry out the randomization and were responsible for participant allocation.

In this trial, only hand-mixed GICs were used and the materials were prepared according to the manufacturer's instructions (powder/liquid ratio of 1:1). The sealants were applied following the steps proposed by Frencken et al. [1996]. First, the occlusal surface was cleaned with a toothbrush and wet cotton wool pellets. Isolation was obtained with cotton wool rolls and the occlusal surface was conditioned with GIC liquid (polyacrylic acid) for $20 \mathrm{~s}$, rinsed with 3 sequences of wet cotton wool pellets, and dried with 3 sequences of dry cotton wool pellets. The GIC was placed on the occlusal surface and pressed into the pits and fissures using the pressfinger technique [Frencken et al., 1996]. After the first setting of the GIC, the excess of material was removed and the occlusion checked and adjusted. Finally, the sealant was protected with a new layer of petroleum jelly and the children were instructed not to eat for at least $1 \mathrm{~h}$.

Additionally, the schools from the municipality where the treatments were performed were included in an oral health program; therefore, all children included in this research received 
Table 1. Baseline demographics of the participants

\begin{tabular}{ll}
\hline Characteristic & Value \\
\hline Age, years & $7 \pm 0.7$ \\
Sex & $96(51)$ \\
$\quad$ Male & $91(49)$ \\
$\quad$ Female & $93(50)$ \\
Side that received sealants & $94(50)$ \\
$\quad$ Right & \\
Left & $53(28)$ \\
Age at intervention (years) & $50(27)$ \\
6 & $84(45)$ \\
7 & \\
8 & $4.08 \pm 3.09$ \\
Caries experience & \\
Baseline DMFT/dmft & $16(8)$ \\
Operator & $15(8)$ \\
1 & $82(44)$ \\
2 & $74(40)$ \\
3 &
\end{tabular}

Values are presented as means \pm SD or numbers (\%).

brushing instructions and dietary advice every 6 months. A mouth hygienist from the municipality was responsible for all orientation. Children were instructed to brush their teeth 3 times a day using a conventional toothbrush, according to the horizontal technique [Muller-Bolla and Courson, 2013], and 1,000-ppm fluoridated dentifrice.

\section{Evaluation}

The follow-up was carried 3, 6, 12, 18, 24, and 36 months after treatments by one independent trained and calibrated examiner specialist in pediatric dentistry. For the assessment of caries lesions, the examiner was trained according to ICDAS [Ismail et al., 2007] and Nyvad criteria [Nyvad et al., 1999] and followed the same training given to the operators of this study. The examiner was also trained according to the following criteria for sealant retention: 0 , fully retained sealant; 1 , partially retained sealant; and 2 , sealant absent [Oba et al., 2009]. This training consisted of $4 \mathrm{~h}$ of specific training and calibration with a "benchmark examiner" using clinical slides, followed by hands-on training that included the evaluation of 20 children who had ART sealants. For the calibration exercises regarding both caries and sealant retention, a total of 65 participants ( $15 \%$ of the sample) were evaluated and reevaluated within a 1 -week interval. The examiner was blinded to the material used and the baseline caries status of participants. The interexaminer $\kappa$ coefficient for the caries lesions assessment was 0.89 , while the intraexaminer $\kappa$ coefficient was 0.94 . For the assessment of sealant retention, $\kappa$ values of 0.97 and 0.98 were obtained for inter- and intraexaminer reproducibility.

Evaluations were also performed on school premises with the aid of a dental probe and mirror. A portable headlight and a manual syringe were used for illumination and for drying tooth surfaces.

Caries-Preventive Effect of ART Sealants
Statistical Analysis

Statistical analysis was carried out using Stata 11.2 software (StataCorp; USA). All significant differences were detected at a 95\% confidence level.

In this research, the tooth was considered an experimental unit and the dependent variable was the survival rate of dentine cavityfree first permanent molars. ICDAS [Ismail et al., 2007] codes 0-4 indicated success, and codes 5 and 6 were considered failures. Kaplan-Meier survival analysis was performed to estimate cumulative survival rates and a log-rank test was used to test for differences in survival rates of dentine cavity-free first permanent molars. The influence of independent variables, such as treatment group (ART sealed/nonsealed molars), jaw (upper/lower), mouth side (right/left), age at treatment $(6,7$, or 8 years old), and caries experience (baseline DMFT/dmft), was evaluated using Cox regression with shared frailty analysis. Additionally, a $\chi^{2}$ test was used to verify the retention of partially and fully retained sealants between the 2 GIC brands used in this study. All of the children who attended at least one of the follow-up evaluations were included in the analyses.

\section{Results}

All 187 children enrolled into the main investigation were also included in this analysis. The demographic baseline data of the participants is described in Table 1.

The percentage of patients who were lost to follow-up after 3 years was $18 \%$. A CONSORT flow diagram of the participants' progress through the trial phases is depicted in Figure 1.

From the 748 permanent molars included in this research, a total of $63(8.4 \%)$ developed a cavitated dentine caries lesion during the 3 -year period of follow-up. Log. rank analysis showed no statistical difference between the first permanent molars that received ART sealants or remained nonsealed for the outcome cavitated dentine caries lesions development (Fig. $2 ; p=0.70$ ). Table 2 depicts information regarding the 3 -year evaluation of the first permanent molars included in this research according to the baseline status of those molars (caries-free occlusal surfaces that were sealed or remained nonsealed and surfaces presenting an enamel lesion at baseline that were sealed or remained nonsealed). The log-rank test was applied to test the hypothesis that surfaces presenting an enamel lesion at baseline would benefit from the application of sealants; however, no difference was found between the groups $(p=0.87)$.

Cox regression with a shared frailty analysis showed that the baseline caries experience was associated with the development of a cavitated dentine caries lesion, indicating that for every 1-unit increase in the baseline DMFT/ $\mathrm{dmft}$ there is a $19 \%$ greater chance of caries lesion devel- 


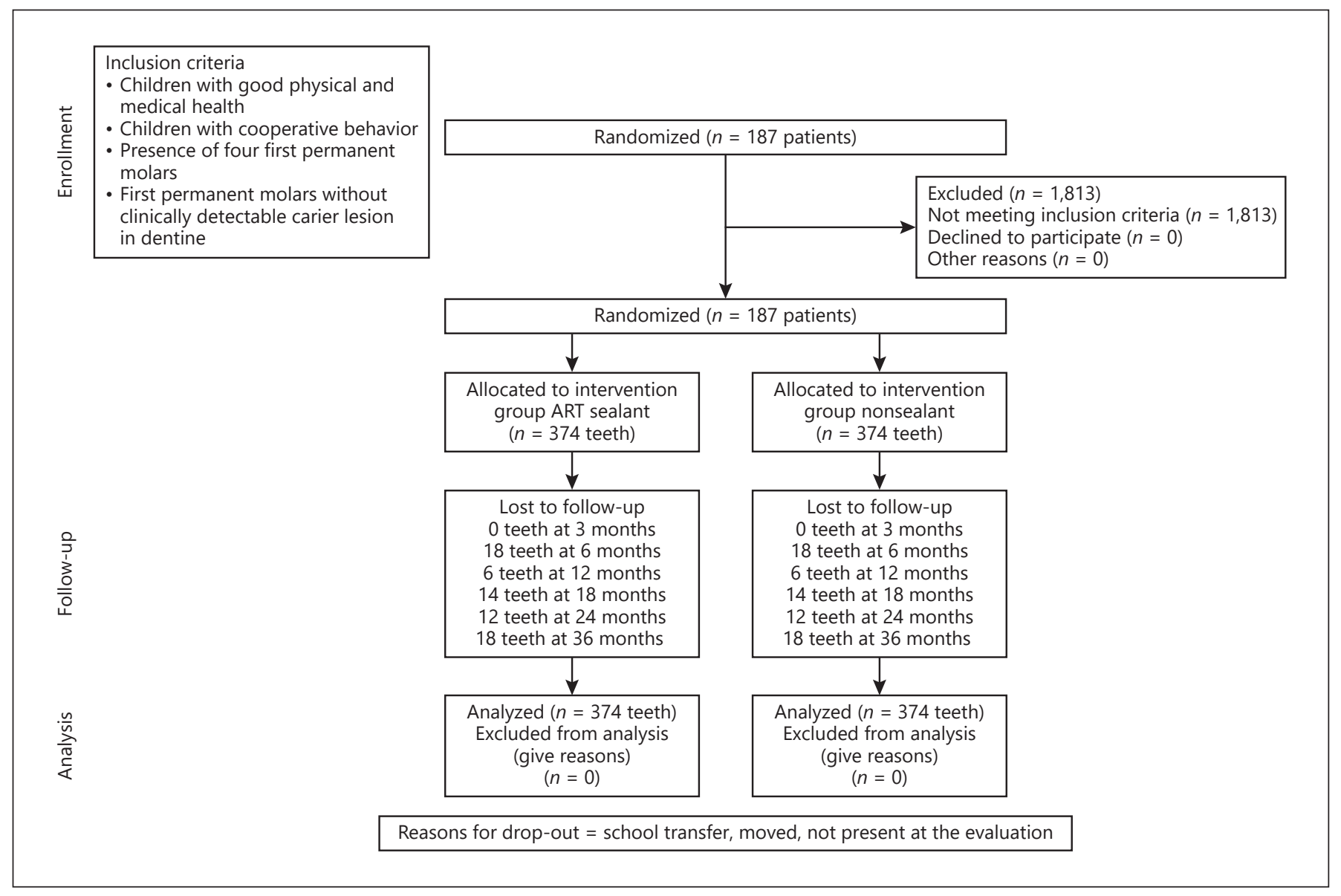

Fig. 1. CONSORT flow diagram of the participants' progress through the trial phases.

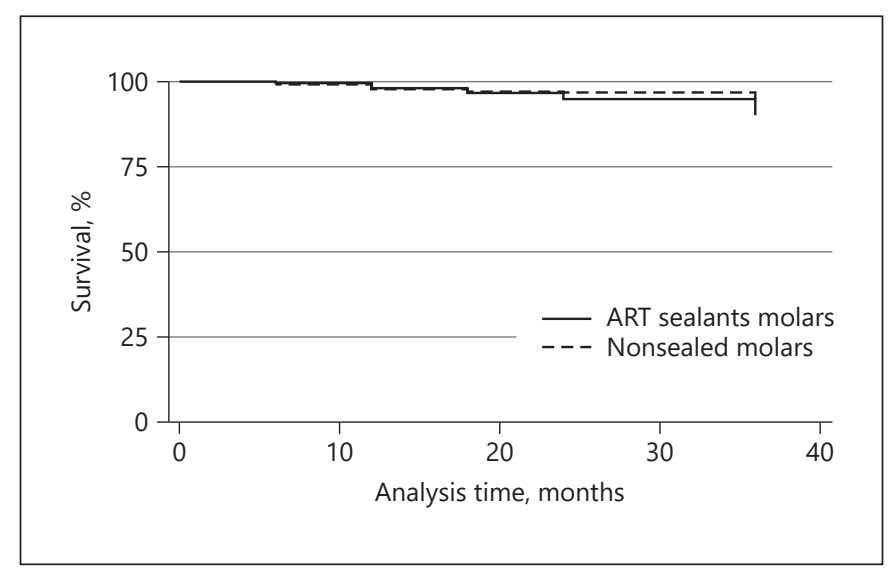

Fig. 2. Survival curves of dentine cavity-free first permanent molars after receiving an ART sealant or remaining nonsealed. Logrank, $p=0.70$. opment in a first permanent molar for both ART-sealed and nonsealed molars (Table 3).

The overall retention of partially and fully retained sealants was $36.7 \%$ after a 3-year follow-up. One GIC brand presented a higher retention of partially and fully retained sealants (Fuji IX: $45.4 \%$ vs. Maxxion R: 25.4\%; $p<0.001$ ); however, neither the GIC brand (log-rank; $p=$ 0.18 ) nor sealant retention had an effect on the cumulative survival rates of dentine cavity-free first permanent molars (log-rank; $p=0.17)$.

\section{Discussion}

\section{Main Findings}

The results of our study showed that there was no difference between molars that received an ART sealant compared to those that remained nonsealed with regards to the development of dentine caries lesions. In addition, 
Table 2. Cavitated dentine caries-free occlusal surfaces in the first permanent molars over the 3-year evaluation

\begin{tabular}{|c|c|c|c|c|c|c|c|c|}
\hline & \multicolumn{2}{|c|}{$\begin{array}{l}\text { No sealant, caries-free } \\
\text { at baseline }\left(n_{\text {baseline }}=275\right)\end{array}$} & \multicolumn{2}{|c|}{$\begin{array}{l}\text { No sealant, caries in enamel } \\
\text { at baseline }\left(n_{\text {baseline }}=99\right)\end{array}$} & \multicolumn{2}{|c|}{$\begin{array}{l}\text { ART sealants, caries-free at } \\
\text { baseline }\left(n_{\text {baseline }}=267\right)\end{array}$} & \multicolumn{2}{|c|}{$\begin{array}{l}\text { ART sealants, caries in enamel } \\
\text { at baseline }\left(n_{\text {baseline }}=107\right)\end{array}$} \\
\hline & $n(\%)$ & SE & $n(\%)$ & SE & $n(\%)$ & SE & $n(\%)$ & SE \\
\hline \multicolumn{9}{|c|}{ Interval, months } \\
\hline 3 & $0(0)$ & - & $0(0)$ & - & $0(0)$ & - & $0(0)$ & - \\
\hline 6 & $1(99.6)$ & 0.4 & $2(97.9)$ & 1.4 & $1(99.6)$ & 0.4 & $1(99.1)$ & 0.9 \\
\hline 24 & $10(96.2)$ & 1.2 & $2(97.9)$ & 1.4 & $13(94.8)$ & 1.4 & $4(95.9)$ & 2.0 \\
\hline 36 & $24(90.1)$ & 1.9 & $6(92.7)$ & 2.9 & $25(89.6)$ & 1.9 & $8(91.0)$ & 3.0 \\
\hline
\end{tabular}

Cumulative percentage survival rates of dentine caries-free occlusal surfaces and SE are shown. $n_{\text {baseline }}=$ number of occlusal surfaces at baseline; $n=$ number of occlusal surfaces that developed cavitated dentine caries lesions.

Table 3. Cox regression with shared frailty analysis of cavitated dentine first permanent molars and associated factors for ARTsealed and nonsealed molars

\begin{tabular}{|c|c|c|}
\hline & HR (95\% CI) & $p$ value \\
\hline $\begin{array}{l}\text { Groups } \\
\text { (ref.: ART sealant) } \\
\text { Nonsealed molars }\end{array}$ & $0.90(0.55-1.49)$ & 0.70 \\
\hline $\begin{array}{l}\text { Jaw } \\
\text { (ref.: upper) } \\
\text { lower }\end{array}$ & $0.85(0.51-1.39)$ & 0.52 \\
\hline $\begin{array}{l}\text { Mouth side } \\
\text { (ref.: right) } \\
\text { left }\end{array}$ & $0.85(0.41-1.65)$ & 0.60 \\
\hline $\begin{array}{l}\text { Age at intervention } \\
\text { (ref.: } 6 \text { years) } \\
7 \text { years } \\
8 \text { years }\end{array}$ & $\begin{array}{l}0.79(0.34-1.80) \\
0.97(0.48-1.97)\end{array}$ & $\begin{array}{l}0.57 \\
0.94\end{array}$ \\
\hline $\begin{array}{l}\text { Caries experience } \\
\text { (baseline DMFT/dmft) }\end{array}$ & $1.19(1.06-1.33)$ & $0.002^{*}$ \\
\hline
\end{tabular}

no statistically significant difference was found between those surfaces that were sound or presented an enamel caries lesion at baseline for both ART sealed and nonsealed groups $(p=0.87)$. This fact reinforces the need to discuss the real indication of pit and fissure sealants [Splieth et al., 2010; Hilgert et al., 2015]. In 2010, Splieth et al. [2010] stated that pit and fissure sealants should be indicated based on an individual caries-risk assessment, surface-specific parameters, caries activity, and patient compliance. Furthermore, sealants applied as preventive measures should be seen as an exception indicated only for patients with a high caries risk and with limited access to health care. In our study, an individual assessment of the caries risk was not performed; but the children included in this trial belonged to a low-income population, presented a high caries experience (mean DMFT/dmft = 4.08) and had limited access to health care, factors that have driven the indication of sealants as a main tool for the prevention and management of occlusal caries lesions [Griffin et al., 2016; Wright et al., 2016]. In this way, we hope that the results of our investigation might increase the awareness to the fact that the application of an ART sealant should not be considered as an isolated preventive or therapeutic measure but it should actually be applied as part of the so-called basic package of oral care, which considers ART sealants as part of preventive measures, combined with instructions for oral hygiene, the use of fluoride, and information regarding dietary patterns [Frencken et al., 2002].

The extent to which the application of sealants can benefit patients in terms of economic implications has been subject of debate. A systematic review of the literature concluded that the cost-effectiveness of sealants application depends on the conditions of delivery [Akinlotan et al., 2018]. This review included a study that demonstrated that a school-based dental sealant program was a more cost-effective approach than no sealants in preventing decay [Zabos et al., 2002]; however, concerns have been raised regarding the treatment effect, since the analysis was not corrected for discrepan- 
cies in caries prevalence among intervention and control groups. Likewise, Griffin et al. [2002] investigated the cost-effectiveness of 3 sealant strategies of first permanent molars (sealants application based on caries risk, sealing all molars or leaving molars unsealed). The results showed that sealing all molars was more costeffective in preventing caries development than the other 2 strategies; however, when considering costs and effectiveness components separately, the sealant application based on caries risk was the least costly and averted more cavitation compared to leaving all molars unsealed. In our study, we did not carry out an economic analysis; nevertheless, since no difference was found in the development of dentine caries lesions, we wonder whether the application of an ART sealant would result in a cost-effective strategy when applied as the main toll for caries prevention.

It is important to highlight that $8.4 \%$ of the first permanent molars included in our research developed a cavitated dentine caries lesion within the 3 years of followup; therefore, it is clear that the preventive program offered to those children, which consists of toothbrushing instructions and dietary counselling given once every 6 months, was somehow not enough to improve the oral health habits to an extent that those children could have been transferred to a healthy status. One can advocate that a daily supervised toothbrush strategy could be seen as the ideal scenario for preventing caries lesions development in high-caries-risk children or occlusal surfaces; however, the high costs related to the salary of personnel [Goldman et al., 2017] can jeopardize the implementation of this type of preventive program. Also, it can be discussed whether the benefits and costs of a daily supervised toothbrushing program can be directly compared with those of a sealant application due to the different nature of both approaches. On the top of that, some concerns remain with regards to the magnitude of the effectiveness of supervised toothbrushing [Dos Santos et al., 2018]. Therefore, it is clear that it is necessary to investigate the best recall interval when using this strategy and, more importantly, to intensify the implementation of individualized dental care based not only on the caries experience and status but also on the oral hygiene and compliance of parents, since it seems that the benefits of individualized dental care programs compensate any additional costs that may be accounted into such strategies [Vermaire et al., 2014].

Interestingly, our results showed an association between the caries experience and the development of cavitated dentine lesions in permanent molars, with partici- pants presenting around $20 \%$ greater chance of developing a cavitated dentine lesion in a first permanent molar for every 1 -unit increase in the baseline DMFT/dmft. Curiously, a similar trend was observed by Heyduck et al. [2006], who stated that sealants seem to be an appropriated preventive measure in low- or moderate-caries-risk individuals. According to the authors, high-caries-risk individuals do not benefit when pit and fissure sealants are applied as the main caries-preventive measure. Still according to the authors, those individuals should be enrolled in an intensive preventive program next to the sealant application.

Further, we noticed that the preventive effect of GIC sealants was not associated with retention of the material. In fact, one systematic review showed that retention of sealants cannot be considered a valid predictor to caries progression outcome in permanent molars [Mickenautsch and Yengopal, 2013]. So, the simple fact that the sealants did not present a high retention rate cannot be seen as a contraindication for the use of this caries-preventive approach.

\section{Study Design}

The split-mouth design of this study enabled the comparison of ART sealants and nonsealed molars in the same individual. Therefore, the exact same conditions related to the caries risk and habits of participants were present for both ART-sealed and nonsealed molars. As a result, the error variance of the study is reduced and an increased statistical power is achieved [Hujoel and DeRouen, 1992]. The cross-over effect among the 2 groups could be seen as a disadvantage inherent to this study design, as it is known that the GIC can release and even recharge from external sources of fluoride [Wiegand et al., 2007]. Although this characteristic is claimed to be beneficial in arresting caries lesions around restorations [Raggio et al., 2016] and preventing cavitated lesion development on the surface where the GIC is applied [de Amorim et al., 2012; Hilgert et al., 2015], a recent systematic review showed that GIC do not promote the arrestment of caries lesions on adjacent surfaces of restored teeth [Tedesco et al., 2016]. Consequently, a cross-over effect on the contralateral molars due to the fluoride release from ART sealants is not expected in our study.

Blinding of operators was not possible in this study. The children and caregivers, as well as the evaluator, were blinded to the commercial brand of the GIC; however, blinding to ART sealed and nonsealed molars was not possible. During the later follow-ups, a considerable number of sealants could not be clinically detected, so we
18

Caries Res 2021;55:12-20 DOI: $10.1159 / 000506466$
Hesse/Guglielmi/Raggio/Bönecker/ Mendes/Bonifácio 
could consider the evaluator as reasonably blind. Additionally, a small drop-out rate (18\%) was observed. Hence, we believe that a relevant validity was achieved in this study. Another limitation to be discussed is the fact that exams were carried out in field conditions and no professional plaque removal was performed prior clinical examination; therefore, it is arguable whether some initial lesions in enamel or underlying dark shadow in dentine (ICDAS 1-4) were overlooked. So, in order to avoid overestimation of the results, the cut-off point of failure of the present investigation was set on the development of cavitated dentine caries lesions (ICDAS 5 and 6), which can be considered a more robust outcome, and enables comparison with other investigations of a similar nature [Hilgert et al., 2015].

The schoolchildren participated in an oral health program provided by the municipality, which comprised toothbrushing instructions including the recommendation of using fluoride toothpaste and dietary advice every 6 months, which could be seen as a nonideal situation as the entire family should be educated towards oral health [Castilho et al., 2013]. The parents were invited to participate in a health educational program; however, the vast majority of them declined to participate. In addition, the children were taught to brush their teeth using a conventional toothbrush with horizontal scrubbing, which can be seen as a nonsatisfactory technique for plaque removal of occlusal surfaces of permanent molars [Frazão, 2011]. These factors could be seen as limitations of our study; however this scenario is still the reality faced by many populations and in fact more effort still needs to be employed in the development of oral health programs that include the entire family and employ brushing techniques that can be more effective in plaque removal, helping parents to achieve and maintain the oral health of their children [Castilho et al., 2013].

In conclusion, the results of the present study show that the application of ART sealants was not more effective in reducing dentine caries lesion development in first permanent molars compared to nonsealed molars.

\section{Acknowledgement}

We would like to thank the schoolchildren, their parents, and the staff of the schools and the health service of Barueri, Brazil, especially Alberto Luiz Ferreira Kesselring, Andrei Alvaro Barrichello Chaves, and Fernanda Michelotti Cajado, for their kind assistance in conducting this study. We are very grateful to Malou Schoenmakers, Nicole Sluitman, Kristel Filius, and Marieke Herman for their dedication during the operative phase. We would like to express our gratitude to GC Europe and FGM Brazil for kindly donating the materials used in this study. We would like to thank the Coordenação de Aperfeiçoamento de Pessoal de Nível Superior (CAPES), the Fundação de Amparo à Pesquisa do Estado de São Paulo (FAPESP), and the Conselho Nacional de Pesquisa (CNPq). We would like to thank the participants of the Research Meetings on Cariology and Pediatric Dentistry from the Academisch Centrum Tandheelkunde Amsterdam (ACTA) for their critical comments.

\section{Statement of Ethics}

This study was approved by the local research ethics committee (protocol 190/08; School of Dentistry, University of São Paulo, Brazil). The parents and/or legal guardians of the subjects were informed about the investigation and treatments and written consent was obtained.

\section{Disclosure Statement}

The funders and manufactures had no role in study design, data collection and analysis, the decision to publish, or the preparation of this paper.

\section{Funding Sources}

This study was partially supported by the Conselho Nacional deDesenvolvimentoCientíficoeTecnológico(CNPq;472882/20104). M.J.S.B. and D.P.R. received a research productivity scholarship from CNPq (305323/2016-4 and 302192/2018-2).

\section{Author Contributions}

D.P.R, M.J.S.B., and C.C.B.: study conception and design. D.H. and C.A.B.G.: data collection. D.H. and F.M.M.: data analysis. D.H., C.A.B.G., D.P.R., M.J.S.B., F.M.M., and C.C.B.: manuscript of this paper.

References

Ahovuo-Saloranta A, Forss H, Walsh T, Nordblad A, Mäkelä M, Worthington HV: Pit and fissure sealants for preventing dental decay in permanent teeth. Cochrane Database Syst Rev. 2017 Jul 31;7:CD001830.

Akinlotan M, Chen B, Fontanilla TM, Chen A, Fan VY. Economic evaluation of dental sealants: A systematic literature review. Community Dent Oral Epidemiol. 2018 Feb;46(1): 38-46.

Carvalho JC, Dige I, Machiulskiene V, Qvist V, Bakhshandeh A, Fatturi-Parolo C, et al. Occlusal Caries: biological approach for its diagnosis and management. Caries Res. 2016; 50(6):527-42. 
Carvalho JC, Thylstrup A, Ekstrand KR. Results after 3 years of non-operative occlusal caries treatment of erupting permanent first molars. Community Dent Oral Epidemiol. 1992 Aug; 20(4):187-92.

Castilho AR, Mialhe FL, Barbosa TS, PuppinRontani RM. Influence of family environment on children's oral health: a systematic review. J Pediatr (Rio J). 2013 Mar-Apr;89(2): $116-23$.

Choo A, Delac DM, Messer LB. Oral hygiene measures and promotion: review and considerations. Aust Dent J. 2001 Sep;46(3):166-73.

Colombo AP, Tanner AC. The Role of Bacterial Biofilms in Dental Caries and Periodontal and Peri-implant Diseases: A Historical Perspective. J Dent Res. 2019 Apr;98(4):373-85.

de Amorim RG, Leal SC, Frencken JE. Survival of atraumatic restorative treatment (ART) sealants and restorations: a meta-analysis. Clin Oral Investig. 2012 Apr;16(2):429-41.

Dos Santos AP, de Oliveira BH, Nadanovsky P. A systematic review of the effects of supervised toothbrushing on caries incidence in children and adolescents. Int J Paediatr Dent. 2018 Jan; 28(1):3-11.

Frazão P: Effectiveness of the bucco-lingual technique within a school-based supervised toothbrushing program on preventing caries: A randomized controlled trial. BMC Oral Health. 2011 Mar;11:11.

Frencken JE, Holmgren C, van Palestein Helderman W. WHO Basic Package of Oral Care (BPOC). Nijmegen, Netherlands: WHO Collaborating Centre for Oral Health Care Planning and Future Scenarios, University of Nijmegen; 2002.

Frencken JE, Pilot T, Songpaisan Y, Phantumvanit P. Atraumatic restorative treatment (ART): rationale, technique, and development. J Public Health Dent. 1996;56(3 Spec No):135-40.

Goldman A, Leal SC, de Amorim RG, Frencken JE. Treating High-Caries Risk Occlusal Surfaces in First Permanent Molars through Sealants and Supervised Toothbrushing: A 3-Year Cost-Effective Analysis. Caries Res. 2017; 51(5):489-99.

Griffin SO, Griffin PM, Gooch BF, Barker LK Comparing the costs of three sealant delivery strategies. J Dent Res. 2002 Sep;81(9):641-5.

Griffin S, Naavaal S, Scherrer C, Griffin PM, Harris $\mathrm{K}$, Chattopadhyay S. School-based dental sealant programs prevent cavities and are cost-effective. Health Aff (Millwood). 2016 Dec;35(12):2233-40.
Hesse D, Bonifácio CC, Guglielmi CA, Franca C, Mendes FM, Raggio DP. Franca Cd, Mendes FM, Raggio DP: Low-cost glass ionomer cement as ART sealant in permanent molars: a randomized clinical trial. Braz Oral Res. 2015; 29(1):pii:S1806-83242015000100261.

Heyduck C, Meller C, Schwahn C, Splieth CH. Effectiveness of sealants in adolescents with high and low caries experience. Caries Res. 2006;40(5):375-81.

Hilgert LA, Leal SC, Mulder J, Creugers NH, Frencken JE. Caries-preventive Effect of Supervised Toothbrushing and Sealants. J Dent Res. 2015 Sep;94(9):1218-24.

Hujoel PP, DeRouen TA. Validity issues in splitmouth trials. J Clin Periodontol. 1992 Oct; 19(9 Pt 1):625-7.

Ismail AI, Sohn W, Tellez M, Amaya A, Sen A, Hasson $\mathrm{H}$, et al. The International Caries Detection and Assessment System (ICDAS): an integrated system for measuring dental caries. Community Dent Oral Epidemiol. 2007 Jun; 35(3):170-8

Jackson RJ, Newman HN, Smart GJ, Stokes E, Hogan JI, Brown C, et al. The effects of a supervised toothbrushing programme on the caries increment of primary school children, initially aged 5-6 years. Caries Res. 2005 Mar-Apr; 39(2):108-15

Mejàre I, Källestål C, Stenlund H, Johansson H. Caries development from 11 to 22 years of age: a prospective radiographic study. Prevalence and distribution. Caries Res. 1998;32(1): 10-6.

Mickenautsch S, Yengopal V. Retention loss of resin based fissure sealants - a valid predictor for clinical outcome? Open Dent J. 2013 Aug; 7(1):102-8.

Mickenautsch S, Yengopal V. Caries-preventive effect of high-viscosity glass ionomer and resin-based fissure sealants on permanent teeth: A systematic review of clinical trials. PLoS One. 2016 Jan;11(1):e0146512.

Monse B, Heinrich-Weltzien R, Mulder J, Holmgren C, van Palenstein Helderman WH. Caries preventive efficacy of silver diammine fluoride (SDF) and ART sealants in a schoolbased daily fluoride toothbrushing program in the Philippines. BMC Oral Health. 2012 Nov; 12(1):52.

Muller-Bolla M, Courson F. Toothbrushing methods to use in children: a systematic review. Oral Health Prev Dent. 2013;11(4):3417.

Nyvad B, Machiulskiene V, Baelum V. Reliability of a new caries diagnostic system differentiating between active and inactive caries lesions. Caries Res. 1999 Jul-Aug;33(4):252-60

Oba AA, Dülgergil T, Sönmez IS, Doğan S. Comparison of caries prevention with glass ionomer and composite resin fissure sealants. J Formos Med Assoc. 2009 Nov;108(11):844-8.
Pandis N, Chung B, Scherer RW, Elbourne D, Altman DG. CONSORT 2010 statement: extension checklist for reporting within person randomised trials. BMJ. 2017 Jun;357:j2835.

Poklepovic T, Worthington HV, Johnson TM, Sambunjak D, Imai P, Clarkson JE, et al. Interdental brushing for the prevention and control of periodontal diseases and dental caries in adults. Cochrane Database Syst Rev. 2013 Dec;18(12):CD009857.

Raggio DP, Tedesco TK, Calvo AF, Braga MM. Do glass ionomer cements prevent caries lesions in margins of restorations in primary teeth?: A systematic review and meta-analysis. J Am Dent Assoc. 2016 Mar;147(3):17785.

Splieth CH, Ekstrand KR, Alkilzy M, Clarkson J, Meyer-Lueckel H, Martignon S, et al. Sealants in dentistry: outcomes of the ORCA saturday afternoon symposium 2007. Caries Res. 2010; 44(1):3-13

Tedesco TK, Bonifácio CC, Calvo AF, Gimenez T, Braga MM, Raggio DP. Caries lesion prevention and arrestment in approximal surfaces in contact with glass ionomer cement restorations - A systematic review and meta-analysis. Int J Paediatr Dent. 2016 May;26(3):161-72.

van't Hof MA, Frencken JE, van Palenstein Helderman WH, Holmgren CJ. The atraumatic restorative treatment (ART) approach for managing dental caries: a meta-analysis. Int Dent J. 2006 Dec;56(6):345-51.

Vermaire JH, van Loveren C, Brouwer WB, Krol $\mathrm{M}$. Value for money: economic evaluation of two different caries prevention programmes compared with standard care in a randomized controlled trial. Caries Res. 2014;48(3):24453.

Wiegand A, Buchalla W, Attin T. Review on fluoride-releasing restorative materials-fluoride release and uptake characteristics, antibacterial activity and influence on caries formation. Dent Mater. 2007 Mar;23(3):343-62.

Wright JT, Crall JJ, Fontana M, Gillette EJ, Nový BB, Dhar V, et al. Evidence-based clinical practice guideline for the use of pit-and-fissure sealants: A report of the American Dental Association and the American Academy of Pediatric Dentistry. J Am Dent Assoc. 2016 Aug;147(8):672-682.e12.

Zabos GP, Glied SA, Tobin JN, Amato E, Turgeon $\mathrm{L}$, Mootabar RN, et al. Cost-effectiveness analysis of a school-based dental sealant program for low-socioeconomic-status children: a practice-based report. J Health Care Poor Underserved. 2002 Feb;13(1):38-48. 